\title{
Personality Disorders in Brazilian Homeless Compared with Non-Psychiatric and Psychiatric Patients
}

\author{
Daniel Bartholomeu1, José M. Montiel1', Geraldo A. Fiamenghi Jr.1, Lucas F. Carvalho², \\ Mara Rubia C. A. Orsini ${ }^{3}$, Cecilia R. Ribeiro ${ }^{4}$ \\ ${ }^{1}$ Postgraduate Department of Educational Psychology, UNIFIEO, Osasco, Brasil \\ ${ }^{2}$ Departament of Psychology, USF, Itatiba, Brasil \\ ${ }^{3}$ Departament of Psychology, Universidade Federalde Goiás, Goiânia, Brasil \\ ${ }^{4}$ UFR d'études psychanalytiques, Université Paris Diderot 7, Paris, France, \\ Email: d bartholomeu@yahoo.com.br, geraldoafj@gmail.com, lucas@labape.com.br, \\ mararubia.mr@gmail.com, cecilia.rodrigues@etu.univ-paris-diderot.fr
}

Received 19 May 2015; accepted 26 June 2015; published 29 June 2015

Copyright @ 2015 by authors and Scientific Research Publishing Inc.

This work is licensed under the Creative Commons Attribution International License (CC BY).

http://creativecommons.org/licenses/by/4.0/

(c) (i) Open Access

\begin{abstract}
Personality disorders are regarded as conditions that involve a maladaptive personality functioning. Homelessness is a worldly debated phenomenon. The present study aimed to understand the situation of homelessness related to the health sector, instead of considering it only as a social or economic problem. Research was conducted with three different groups, male and female, living in a Metropolitan area of Southeast Brazil, the first composed of $\mathbf{7 1}$ homeless people; the second, 74 psychiatric patients; and the third, 250 college students that completed the Personality Disorders Dimensional Inventory (IDTP). A logit regression model and ROC curve were used to analyse data. Results showed that homeless people scored higher in all dimensions, especially Antisocial and Schizotypal, compared with the other groups. Although the number of participants was reduced to only one part of the country, as well as the correlational design preventing clearer causal inferences, the results of this research call the attention for the need of new investigations to homeless people's mental health, aiming to focus on public health policies that could deal with the problem.
\end{abstract}

\section{Keywords}

Homelessness, Personality Disorders, Mental Health, Health Policies 


\section{Introduction}

Personality disorders are regarded as conditions involving a maladaptive personality functioning. According to classification manuals, especially DSM-V (APA, 2013), the most important semiotic feature of those disorders consists in a persistent pattern of an emotional and behavioral experience significantly deviant from the expected, causing damage to interpersonal, social, cognitive, affective and occupational aspects. Such description, polemic as it might be theoretically, is not as simple to work in practice, despite being relevant as a diagnosis throughout history.

This definition also includes social problems derived from the disorder, for the people suffering from it, as well as the nearest interpersonal relations, and society in general (Coid et al., 2006). In fact, one of the pathognomonic descriptors for the incidence of a personality disorder is related to incidence, duration, and social consequences caused by that lack of adaptation.

Hence, many studies (Koegel et al., 1988; Ball et al., 2005; Coid et al., 2006; Maguire et al., 2009; Cockersell, 2011) emphasize the need for amplifying the associations between a personality disorder and demographic aspects such as the patient's socioeconomic status. It has to be noted that a social risk situation may be intimately related to general psychopathology as both can cause, result, or intensify each other. Studies especially point to data related to homelessness as a strong social context relating mental illness and socioeconomic impact.

Homelessness is a worldly debated phenomenon. The term "homeless" refers to someone who lives on the streets, or in places that are insufficient or out of standards from what is called human habitation, such as abandoned buildings, for example. However, other people at risk, such as the ones who spend more than 50\% of their earnings with home expenses (without taking in account other expenses, such as food, or house maintenance), as well as those who live in relatives' houses (normally sleeping in their couches) are also targets of social attention because they are in process of homelessness (Canadian Institute for Health Information, 2007).

Maguire et al. (2009) state that homelessness results of a complicated interaction between socio environmental factors with mental health ones. Chronic poverty, characterized by a context of lack of accommodation could generally be associated to a psychiatric disorder, elucidating the relationship between eviction and a history of psychopathology.

Considering homelessness as part of a health issue opens a new perspective to understanding that population, as they are not anymore a result of purely socioeconomic questions, but a consequence of a psychological disorder as well. Cockersell (2011) writes that such a social problem is more a symptomatological manifestation of a mental disorder than a causal result of it. In other words, the author states that a person not having a home is a pathoplastic expression of a psychological disease.

Not having a home might conduct to the development of a pathology. That does not mean that a homeless person has a mental disease, but being a homeless makes that person more prone to developing or enhancing pathologies. Constant stress, inability to cope with borderline situations, lack of social support, low self-esteem, and suicidal behaviour are typical of that population, increasing the prognosis, as well as maintaining the homeless condition (Canadian Institute for Health Information, 2007).

We do not intend to forget all of the factors present in the problem, but to understand that vulnerability to be homeless might be related to the existence of a personality disorder as well. There are other social and economic questions related to the lack of housing accommodation. However, a shorter homelessness situation could be caused by transient problems, such as being sacked from work, lack of family support, concentration in studies, investment in less assertive areas. On the other hand, a more permanent homelessness situation could be associated to a psychological diagnosis, especially, psychosis, depression, substance use and a Personality disorder (Caton et al., 2005).

Correlation studies on homelessness and Personality disorders are still limited due to comorbidity with other substance use disorders, such as alcohol, cannabis, and cocaine. Nevertheless, a research by Ball et al. (2005) showed important results concerning Personality disorders: $88 \%$ of the population studied (homeless people assisted in a Manhattan support centre) were diagnosed with at least one sort of Personality disorder, the most common being paranoid personality, followed by schizoid. On the other hand, borderline and antisocial disorders were strongly correlated with substance use, as $74 \%$ of the sample was diagnosed as such. Finally, avoidant, and especially obsessive-compulsive disorders were diagnosed in $85 \%$ of the sample.

Due to the high incidence of that diagnosis in homeless people shown in research around the world, as well as due to the high social impact stemmed from that context, it is relevant to identify the situation in Brazil. Such studies will bring new perspectives to aid that group, not only in social, but also in health areas. The exact order 
of events, that is, whether homelessness depicts a symptomatological manifestation of a psychiatry disorder, or if social issues have started or intensified the development of such a condition, is irrelevant, as data relate the presence of a symptomatology to homelessness.

There is an estimate of around 1\% of the population being homeless (more than 1 million people) in Brazil. Even if that population is not able to look for help or follow a treatment, they are part of a target group for intervention, as being at high risk for comorbidity. Moreover, if those people looked for professional support, social system would not be prepared to embrace the complexity of the situation, as it is normally thought of as a purely economic and housing one.

Thus, the present study aimed to understand the situation of homelessness as related to health sector, instead of considering it only as a social or economic problem. The prognosis could be more positive if the problem were more widely assumed in all of its dimensions, and developing health public policies to deal with it.

\section{Method}

\subsection{Participants}

Research was Conducted with three Different Groups

The first group consisted of 71 homeless people, living in a Metropolitan area of Southeast Brazil, aged 18 to 68 years, $90.7 \%$ males and $9.3 \%$ females, $90.0 \%$ reported not taking any medicines, and $100 \%$ have not finished primary school, although there were no illiterate subjects. The majority of participants were homeless either because as adolescents they had ran away from home due to risky situations, such as abusive parents and maltreatment (66\%), or because they were expelled from their homes as a result of drug use or antisocial behaviour (34\%). All participants were attended at an institution that provided food (breakfast and lunch), clothing, shower, and a dormitory for homeless and itinerant people. Social workers at the institution tried to send the homeless to other government organisms, aiming to provide bus fares or job opportunities. At the time of the research, all of the homeless were unemployed.

The second group consisted of psychiatric patients $(\mathrm{N}=74)$, aged 18 to 67 years, $54(73 \%)$ female, who attended a psychiatric unit. School attendance varied from unfinished primary school to college degree. All of the participants in the group were treated with medication and 56 had a known diagnosis.

The third group, non-patients, consisted of 250 college students, none of them in psychiatric treatment or using medication, aged 18 to 67 years, 74 male and 176 females.

\subsection{Instrument}

Data were collected using the Personality Disorders Dimensional Inventory (IDTP, Carvalho, 2008). This instrument was chosen firstly because it was created and adapted to Brazilian culture and reality, and secondly because it allowed an assessment of a profile tendency of personality disorders in homeless people. IDTP is an instrument to assess Personality Disorders using 100 items, grouped in 15 different scales: Depressive (Scale I), Schizoid (Scale II), Borderline (Scale III), Paranoid (Scale IV), Sadistic (Scale V), Compulsive (Scale VI), Masochist (Scale VII), Antisocial (Scale VIII), Dependent (Scale IX), Histrionic (Scale X), Negativist (Scale XI), Schizotypal (Scale XII), Avoidant (Scale XIII), Narcissistic (Scale XIV), Social Desirability (Scale XV, which was excluded from the analysis). Responses were distributed in a Likert scale with 4 points, being: (1) It does not describe me; (2) It describes me a little; (3) It describes me a lot; (4) It totally or extremely describes me.

\subsection{Procedure}

The first group (homeless people) completed the inventory individually in an assisted fashion, because they presented low literacy abilities, as well as understanding difficulties due to substance use (alcohol, cocaine, crack, cannabis), although none of them were under influence when assessed. Nevertheless, application time exceeded estimated 20 minutes. The second group (psychiatric patients) was individually assessed in the psychiatric unit they were attended and the third group was collectively assessed in college settings. Participants signed a letter of consent and the University's Ethics Committee approved the research (document number 2342/2011)

\subsection{Results}

According to the aims of this study, analysis was firstly conducted using repeated means, for the three groups, (homeless, psychiatric patients and non-patients) in all 14 IDTP dimensions. Scale XV (Social Desirability) was 
not used due to the objectives of this study. Table 1 shows descriptive statistics for the groups in all dimensions, and the group that presented the highest means is in italics.

According to the table, homeless group presented the highest means in all IDTP dimensions. The group of non-patients presented the lowest scores in all situations. Null hypothesis was verified through significance test ( $\mathrm{F}=10.908 ; p<0.01)$, suggesting a difference among the groups profiles in IDTP dimensions.

Particularly, it is remarkably to observe the elevations in Borderline and Compulsive dimensions. However, this seemed to be a tendency for the whole of the sample, meaning elevation in both dimensions. Specifically concerning the homeless group profile, the most visible discrepancies related to other groups was an elevation in Antisocial and Schizotypal dimension and lowering in Dependent dimension.

Continuing analysis, and trying to validate the predictive capacity of IDTP dimensions in groups, two logit regression analysis were conducted to predict for the homeless and non-patients groups, and homeless and psychiatric patients. Table 2 allows observing which IDTP dimensions stayed within the predictive model, as well as their weight in it.

Table 1. Descriptive data in groups in IDTP dimensions.

\begin{tabular}{|c|c|c|c|c|c|c|c|}
\hline Dimensions & Groups & M & $\mathrm{DP}$ & Dimensions & Groups & M & DP \\
\hline \multirow{3}{*}{ Depressive } & Homeless & 2.39 & 0.74 & \multirow{3}{*}{ Antisocial } & Homeless & 2.33 & 0.59 \\
\hline & Non-Patients & 1.40 & 0.49 & & Non-Patients & 1.70 & 0.49 \\
\hline & Psychiatric & 2.12 & 0.81 & & Psychiatric & 1.85 & 0.68 \\
\hline \multirow{3}{*}{ Schizoid } & Homeless & 2.20 & 0.67 & \multirow{3}{*}{ Dependent } & Homeless & 1.92 & 0.67 \\
\hline & Non-Patients & 1.46 & 0.43 & & Non-Patients & 1.50 & 0.49 \\
\hline & Psychiatric & 1.95 & 0.75 & & Psychiatric & 1.85 & 0.63 \\
\hline \multirow{3}{*}{ Borderline } & Homeless & 2.85 & 0.60 & \multirow{3}{*}{ Histrionic } & Homeless & 2.20 & 0.68 \\
\hline & Non-Patients & 2.44 & 0.57 & & Non-Patients & 1.72 & 0.55 \\
\hline & Psychiatric & 2.67 & 0.74 & & Psychiatric & 1.85 & 0.71 \\
\hline \multirow{3}{*}{ Paranoid } & Homeless & 2.65 & 0.56 & \multirow{3}{*}{ Negativist } & Homeless & 2.24 & 0.66 \\
\hline & Non-Patients & 1.78 & 0.47 & & Non-Patients & 1.39 & 0.37 \\
\hline & Psychiatric & 2.16 & 0.76 & & Psychiatric & 1.77 & 0.65 \\
\hline \multirow{3}{*}{ Sadistic } & Homeless & 1.85 & 0.69 & \multirow{3}{*}{ Schizotypal } & Homeless & 2.57 & 0.76 \\
\hline & Non-Patients & 1.49 & 0.47 & & Non-Patients & 1.75 & 0.64 \\
\hline & Psychiatric & 1.68 & 0.64 & & Psychiatric & 2.01 & 0.78 \\
\hline \multirow{3}{*}{ Compulsive } & Homeless & 3.07 & 0.69 & \multirow{3}{*}{ Evitative } & Homeless & 2.46 & 0.70 \\
\hline & Non-Patients & 2.77 & 0.64 & & Non-Patients & 1.71 & 0.51 \\
\hline & Psychiatric & 2.89 & 0.75 & & Psychiatric & 2.15 & 0.78 \\
\hline \multirow{3}{*}{ Masochist } & Homeless & 2.38 & 0.69 & \multirow{3}{*}{ Narcissistic } & Homeless & 2.46 & 0.59 \\
\hline & Non-Patients & 1.51 & 0.51 & & Non-Patients & 2.19 & 0.51 \\
\hline & Psychiatric & 1.93 & 0.66 & & Psychiatric & 2.23 & 0.63 \\
\hline
\end{tabular}


Table 2. Logit regression model with homeless and non-patients.

\begin{tabular}{ccccccc}
\hline & B & S.E. & Wald & df & $p$ & Exp(B) \\
\hline Depressive & -1.571 & 0.426 & 13.632 & 1 & 0.000 & 0.208 \\
Borderline & 1.281 & 0.452 & 8.026 & 1 & 0.005 & 3.601 \\
Paranoid & -1.946 & 0.440 & 19.555 & 1 & 0.000 & 0.143 \\
Sadistic & 1.200 & 0.446 & 7.256 & 1 & 0.007 & 3.321 \\
Antisocial & -1.033 & 0.435 & 5.637 & 1 & 0.018 & 0.356 \\
Negativist & -2.005 & 0.509 & 15.509 & 1 & 0.000 & 0.135 \\
\hline
\end{tabular}

Dimensions more weighed in the model were Negativist, followed by Paranoid. However, it is possible to note an impressive contribution of all dimensions kept in the model. After refining that information, Table 3 shows data related to percentages of accuracy of IDTP dimensions predictions for both groups.

As observed in the table, IDTP was able to adequate predict $73.2 \%$ of homelessness cases, that is, to identify them as belonging to that group, and, similarly, $96 \%$ of non-patients. Total of accuracy percentage is $90.9 \%$. In the same sense, variance percentage explained by IDTP dimensions was equal to r2Cox \& Snell $=0.44$ e r2Nagelkerke $=0.67$. Based on the regression analysis, a variable was produced, referring to the accuracy probability of relevant dimensions of IDTP on group prediction. That variable was investigated via ROC curve analysis, aiming to knowing its sensitivity and specificity. The area under the curve equals 0.94 , considered a very good result.

Analysis of ROC curve confirms results from logit regression, that is, a predictive ability expressed by 6 IDPT dimensions. In this case, sensitivity equals 1.0 and specificity equals 0.96 .

The same procedure was conducted with homeless and psychiatric patients. Considering this latter group (psychiatric patients) more related to homeless group than non-patients, less expressive predictive results were expected. Table 4 presents which IDTP dimensions were stable in the predictor model and the weight of model variables.

The two dimensions, Paranoid and Antisocial, presented similar weights in the model and were the only significant ones from a total of 14 IDTP dimensions. Despite the expressive contribution of the two dimensions to the model, differently from the data presented previously, the variance percentage explained by the dimensions was smaller ( $r 2$ Cox \& Snell = 0.16; r2Nagelkerke $=0.22$ ).

Table 5 presents data related to accuracy percentages in IDTP dimensions and group prediction. IDTP was able to adequate predict $67.6 \%$ of the cases of homeless, that is, identify them as belonging to that group, and similarly, $70.8 \%$ of psychiatric patients, both lower percentages than those observed with non-patient group. The total accuracy percentage was $69.2 \%$. Likewise, to data using non-patients group, a logit regression variable was generated and used to analyse the ROC curve. The area under the curve, equals 0.75 , lower than data observed with the other group, bordering ideal cut point (0.80)

Analysis of ROC curve reinforces the results of logit analysis, that is, a predictive ability expressed by two IDTP dimensions in differentiating homeless and psychiatric groups. In this case, sensitivity was 0.98 , and specificity was 1.0.

\section{Discussion}

This research was planned due to the lack of studies that deal with Personality disorders affecting homeless people in Brazil. Thus, results suggest changes in Personality dispositions in those people, higher than in psychiatric patients, indicating the acute extension of their problems, as psychiatric patients were cared for in specialized clinical units, opposite to homeless people. In that sense, data suggest that problems related to Personality deviations are more critical in homeless people, requiring attention and care in terms of mental health. Those factors emphasize the need for direct Brazilian public policies aimed at homeless reality, treating the problem within health issues, prior to a process of social inclusion, similarly to other countries, such as USA and United 
Table 3. Accuracy Percentages in prediction of homeless and non-patients.

\begin{tabular}{|c|c|c|c|c|}
\hline \multirow{2}{*}{\multicolumn{2}{|c|}{ Observed Data }} & \multicolumn{3}{|c|}{ Predict } \\
\hline & & \multicolumn{2}{|c|}{ Groups } & \multirow[t]{2}{*}{ Correct \% } \\
\hline & & Homeless & Non-patients & \\
\hline \multirow{3}{*}{ Groups } & Homeless & 52 & 19 & 73.2 \\
\hline & Non-patients & 10 & 237 & 96.0 \\
\hline & & & & 90.9 \\
\hline
\end{tabular}

Table 4. Logit regression model with homeless and psychiatric patients.

\begin{tabular}{rcccccc}
\hline & B & S.E. & Wald & df & $p$ & $\operatorname{Exp}(\mathrm{B})$ \\
\hline Paranoid & -0.711 & 0.301 & 5.570 & 1 & 0.018 & 0.491 \\
Antisocial & -0.843 & 0.329 & 6.562 & 1 & 0.010 & 0.431 \\
\hline
\end{tabular}

Table 5. Accuracy percentages in predictions of homeless and psychiatric patients.

\begin{tabular}{|c|c|c|c|c|}
\hline \multirow{2}{*}{\multicolumn{2}{|c|}{ Observed data }} & \multicolumn{3}{|c|}{ Predict } \\
\hline & & \multicolumn{2}{|c|}{ Groups } & \multirow[t]{2}{*}{ Correct \% } \\
\hline & & Homeless & Psychiatric & \\
\hline \multirow{3}{*}{ Groups } & Homeless & 48 & 23 & 67.6 \\
\hline & Psychiatric & 21 & 51 & 70.8 \\
\hline & $\%$ total & & & 69.2 \\
\hline
\end{tabular}

Kingdom deal with it (Drake et al., 1997; Fazel, Khosia, Doll \& Geddes, 2008).

Therefore, as Caton et al. (2005) observe, it is fundamental that attention to homeless people's mental health precedes social care, for it plays a large role in recovery from the latter. Besides, apart from the mental health problem presented by that population, which was showed in this research, comprehension of main Personality processes allows for the planning of interventions stemming from techniques of emotional regulation, problem solving, and others aiming to care and prevent affective dysregulation and disruptive behaviors (Fazel, Khosia, Doll, \& Geddes, 2008).

Despite all the scores in each disorder being higher than the means of control and clinic groups, higher changes in Personality patterns present in the homeless group were Paranoid, Antisocial, Histrionic and Schizotypal. Those results are close to Fazel et al. (2008), as they also found more cases of antisocial behaviour in homeless people, as well as schizoid, borderline and dependent patterns. Actually, in Borderline and Sadistic Personality predispositions, homeless group scored similarly to psychiatric patients.

\section{Conclusion}

Although the number of participants was restricted to one part of the country, as well as the correlational design preventing more clearly causal inferences, the results of this research call the attention for the need of new investigations concerning homeless people's mental health, aiming to focus on public health policies that could deal with the problem.

\section{References}

APA (2013). Diagnostic and Statistical Manual of Mental Disorders (DSM-V). Arlington, VA: American Psychiatric Association. 
Ball, S. A., Cobb-Richardson, P., Connolly, A. J., Bujosa, C. T., \& O’Neall, T. W. (2005). Substance Abuse and Personality Disorders in Homeless Drop-In Center Clients: Symptom Severity and Psychotherapy Retention in a Randomized Clinical Trial. Comprehensive Psychiatry, 46, 371-379. http://dx.doi.org/10.1016/j.comppsych.2004.11.003

Canadian Institute for Health Information (2007) Improving the Health of Canadians: Mental Health and Homelessness. Ottawa: CIHI.

Carvalho, L. de F. (2008). Construção e validação do Inventário Dimensional dos Transtornos da Personalidade. Master Thesis, Itatiba: Universidade São Francisco. http://www.usf.edu.br/galeria/getImage/385/6974254995417636.pdf

Caton, C. L., Dominguez, B., Schanzer, B., Hasin, D. S., Shrout, P. E., Felix, A., McQuistion, H., Opler, L., \& Hsu, E. (2005). Risk Factors for Long-Term Homelessness: Findings from a Longitudinal Study of First-Time Homeless Single Adults. American Journal of Public Health, 95, 1753-1759. http://dx.doi.org/10.2105/AJPH.2005.063321

Cockersell, P. (2011) Homelessness and Mental Health. Journal of Public Mental Health, 10, 88-98. http://dx.doi.org/10.1108/17465721111154284

Coid, J., Yang, M., Tyrer, P., Roberts, A., \& Ullrich, S. (2006). Prevalence and Correlates of Personality Disorder in Great Britain. British Journal of Psychiatry, 188, 423-431. http://dx.doi.org/10.1192/bjp.188.5.423

Drake, R. E., Yovetich, N. A., Bebout, R. R., Harris, M., \& McHugo, G. J. (1997). Integrated Treatment for Dually Diagnosed Homeless Adults. Journal of Nervous and Mental Disease, 185, 298-305. http://dx.doi.org/10.1097/00005053-199705000-00003

Fazel, S., Khosia, V., Doll, H., \& Geddes, J. (2008). The Prevalence of Mental Disorders among the Homeless in Western Countries: Systematic Review and Meta-Regression Analysis. PlosMedicine, 5, 1670-1681. http://dx.doi.org/10.1371/journal.pmed.0050225

Koegel, P., Burnam, A., \& Farr, R. K. (1988). The Prevalence of Specific Psychiatric Disorders among Homeless Individuals in the Inner City of Los Angeles. Archives of General Psychiatry, 45, 1085-1092. http://dx.doi.org/10.1001/archpsyc.1988.01800360033005

Maguire, N., Johnson, R., Vostanis, P., Keats, H., \& Remington, B. (2009). Homelessness and Complex Trauma: A Review of the Literature. Southampton: University of Southampton. www.personal.soton.ac.uk/nm10/Complex_Trauma.doc 